Revue des patrimoines

\title{
Le souvenir de la Grande Guerre dans les réseaux de conservation de l'Entre-Deux-Guerres. Une préhistoire du Bouclier bleu
}

\section{Pierre Leveau}

\section{OpenEdition}

\section{Journals}

Édition électronique

URL : http://journals.openedition.org/insitu/10903

DOI : 10.4000/insitu.10903

ISSN : 1630-7305

Éditeur

Ministère de la culture

Référence électronique

Pierre Leveau, «Le souvenir de la Grande Guerre dans les réseaux de conservation de l'Entre-DeuxGuerres. Une préhistoire du Bouclier bleu », In Situ [En ligne], 23 | 2014, mis en ligne le 21 février 2014, consulté le 01 mai 2019. URL : http://journals.openedition.org/insitu/10903 ; DOI : 10.4000/ insitu. 10903

Ce document a été généré automatiquement le 1 mai 2019.

\section{(c) $(1) \Theta \theta$}

In Situ Revues des patrimoines est mis à disposition selon les termes de la licence Creative Commons Attribution - Pas d'Utilisation Commerciale - Pas de Modification 4.0 International. 


\title{
Le souvenir de la Grande Guerre dans les réseaux de conservation de l'Entre-Deux-Guerres. Une préhistoire du Bouclier bleu
}

\author{
Pierre Leveau
}

\section{Introduction}

1 Quelles leçons les réseaux de conservation du patrimoine qui ont émergé dans l'EntreDeux-Guerres sous l'égide de la Société des Nations (SDN) ont-ils tirées de la GrandeGuerre? Apparemment, presque aucune: on ne trouve que très peu de textes sur la question dans les archives qu'ils nous ont léguées ${ }^{1}$. Certains de leurs membres, comme Paul Léon, en $1926^{2}$, l'ont posée, mais uniquement sur le plan national et dans le cadre de la commission des Réparations, dont dépendaient les travaux de restauration dans chaque pays ${ }^{3}$. Il convient donc de s'interroger sur le silence des instances internationales sur cette question. Comment expliquer par exemple celui de la SDN ? Par le pacifisme de ses membres et la censure qu'elle choisit d'exercer après-guerre pour désarmer les esprits? Ou par le diagnostic de ses experts sur les causes du vandalisme et de la guerre? L'idée de conflit n'était pas étrangère aux spécialistes du patrimoine, tout pacifistes qu' ils fussent ? Aloïs Riegl avait montré dès 1903 que sa conservation s'appuie sur des stratégies d'alliance analogues à celles développées en temps de guerre ${ }^{4}$ et Max Dvorak proposa à partir de 1916 des méthodes pour le protéger des dommages qu'il subissait durant la Grande Guerre ${ }^{5}$. Si l'effondrement de l'empire austro-hongrois explique l'oubli de ces textes dans l'Entre-Deux-Guerres ${ }^{6}$, les responsables des institutions patrimoniales durent néanmoins résoudre les problèmes qu'ils posèrent: sur quelles valeurs et comment fonder un réseau de conservation à l'épreuve du nihilisme moral et des guerres? Le Comité international du Bouclier bleu répond aujourd'hui à cette question; on rappelle dans ce qui suit quelle fut sa préhistoire, en remontant de la naissance du projet au XIX ${ }^{\mathrm{e}}$ 
siècle jusqu'au déclenchement de la Seconde Guerre mondiale, pour dire quelle leçon les experts de la conservation ont tirée de la Grande Guerre. L'Entre-Deux-Guerres, hanté par son souvenir, se divise de ce point de vue en quatre périodes, allant de la création de la SDN en 1919 jusqu'au pacte Briand-Kellogg pour la paix, qui réintroduit l'Allemagne dans le jeu diplomatique à partir de 1926 ; puis de son intégration à la SDN jusqu'à son départ en 1933, qui fit péricliter la conférence sur le désarmement; de ce tournant ensuite jusqu'à la guerre d'Espagne, qui raviva en 1936 le souvenir de la Der des ders; de cette tragédie enfin, qui sonna finalement le glas de la SDN, jusqu'au déclenchement de la Seconde Guerre mondiale en 1939.

\section{Le projet d'une Croix-Rouge du patrimoine avant la Grande Guerre}

2 Vaut-il mieux sacrifier, en temps de guerre, dix mille œuvres à une vie ou dix mille vies à une œuvre? La question que posa Robert Musil ${ }^{7}$ après la Seconde occupait en fait les sociétés savantes depuis l'Exposition universelle de 1889. Le ministre français du Commerce y avait réuni à Paris, du 24 au 29 juin, en marge du congrès universel pour la Paix, un congrès international de Protection des Monuments et des CEuvres $\mathrm{d}^{\prime} \mathrm{Art}^{8}$ (fig. $^{\circ}$ 1). Après Victor Hugo, son secrétaire général Charles Normand estimait que la beauté est un bien commun de l'humanité et proposait aux gouvernements de désigner en temps de paix les monuments dont la sauvegarde serait assurée en temps de guerre par une convention internationale. Se référant au Comité international de la Croix-Rouge (CICR) fondé à Genève en 1863 , le VI ${ }^{\mathrm{e}}$ vœu qu'il fit adopter par le congrès demandait la création d'un organe semblable pour le patrimoine, visant à protéger les œuvres d'art victimes des conflits armés. Ce vœu fut par la suite repris par les deux conférences pour le Désarmement et la Prévention de la guerre qui se réunirent à La Haye en 1899 et 1907. L'article 27 de la «IVe convention sur les lois et coutumes de guerre sur terre » stipulait ainsi que les monuments historiques, les bibliothèques et les musées seraient épargnés au même titre que les hôpitaux, s'ils n'avaient pas de fonction militaire'. L'article 5 de la « IX ${ }^{e}$ convention sur le bombardement par les forces navales " précisait par ailleurs qu'ils seraient signalés par de grands panneaux rectangulaires divisés suivant la diagonale en deux triangles, noir en haut et blanc en bas ${ }^{10}$. Mais le pavillon prévu par l'article 5 de la IX e convention s'adressait paradoxalement aux forces navales, non terrestres, au tir imprécis. En l'absence de commission autorisée à procéder à des vérifications, cette tentative d'aligner le régime de protection du patrimoine sur celui des hôpitaux n'aboutit pas : la Grande Guerre qui éclata sept ans plus tard n'épargna pas plus les œuvres que les vies humaines. 
Figure 1

\begin{tabular}{|c|c|}
\hline \multicolumn{2}{|c|}{$\begin{array}{l}\text { CONGRĖS INTERNATIONAL POUR LA PROTECTION DES CEUVRES D'ART ET DES } \\
\text { MONUMENTS } \\
\text { Paris, } 24-26 \text { juin } 1889\end{array}$} \\
\hline \multicolumn{2}{|r|}{ Bureau du Comité d’organisation } \\
\hline \multicolumn{2}{|r|}{ Président } \\
\hline Garnier, Ch. & Architecte, membre de l'Institut \\
\hline \multicolumn{2}{|r|}{ Vice-Présidents } \\
\hline Ravaisson, $\mathrm{F}$. & $\begin{array}{l}\text { Membre de l'Institut (Académie des inscriptions et belles-lettre et } \\
\text { Académies des sciences morales et politiques) }\end{array}$ \\
\hline Vitu, A. & $\begin{array}{l}\text { Homme de lettres, vice-président de la Société des Amis des } \\
\text { monuments parisiens. }\end{array}$ \\
\hline \multicolumn{2}{|r|}{ Secrétaire général } \\
\hline Normand, Ch. & $\begin{array}{l}\text { Architecte diplômé par le gouvernement, Directeur de L'ami des } \\
\text { monuments, Secrétaire général de la Société des Amis des } \\
\text { Monuments parisiens }\end{array}$ \\
\hline
\end{tabular}

COMITÉ d'ORgANISATION DU CONgRÈs POUR LA PROTECTION DES œUVRES D'ART ET DES MONUMENTS (1889).

(c) PIERRE LeVEAU.

Fallait-il donc consacrer en temps de paix des conférences à la question de la protection du patrimoine en temps de guerre? Malgré cet échec, le $\mathrm{VI}^{\mathrm{e}}$ vœu de 1889 ne fut pas oublié dans l'Entre-Deux-Guerres. Soutenu par les correspondants suisses du congrès, Théodore de Saussure et Henry de Geymüller ${ }^{11}$, l'historien d'art Ferdinand Vetter en reprit en effet l'idée. Lors d'un symposium sur la protection des monuments réuni à Genève le 20 avril 1915, il la rattacha à celles défendues à La Haye et proposa de créer un organisme chargé de faire respecter l'article 27 de la $\mathrm{VI}^{\mathrm{e}}$ convention de 1905, appelé Croix d'Or, qui se serait installé dans un État neutre, comme le Comité international de la Croix-Rouge ${ }^{12}$. Mais il fallut attendre la fin de la Grande Guerre pour que ces vœux pieux fassent l'objet d'une expertise rompant avec les métaphores poétiques. En avril 1918, le conservateur du Stedelijk Museum de Lakenhal, Jacobus Cornelis Overvoorde, alors président de la Société royale néerlandaise d'Archéologie ${ }^{13}$ (KNOB), en avait soumis l'idée au ministre des Affaires étrangères des Pays-Bas, qui l'avait chargé le 3 mai de composer un comité et de lui remettre un rapport sur la question (fig. $\mathbf{n}^{\circ} \mathbf{2}$ ). La guerre n'était pas encore finie et la SDN n'était pas née, mais l'expérience avait déjà montré que l'article 27 sur la protection des monuments de la $\mathrm{IV}^{\mathrm{e}}$ convention était inapplicable. Proche de l'architecte Joseph Cuypers, J.C. Overvoorde était à la tête d'un vaste réseau dont les membres voulaient tirer les leçons du désastre de la Grande Guerre. À la veille de l'armistice, le gouvernement hollandais leur proposait de préparer le document qu'utiliserait après-guerre la Cour permanente d'Arbitrage de La Haye pour garantir l'efficacité de l'article 27 après la ratification des nouveaux traités de paix. Mais plutôt que de proposer d'autres textes, le comité composé de juristes, de conservateurs, d'architectes et de militaires, choisit de cibler par une série de questions les obstacles qui empêchaient son application pour ouvrir les négociations qui permettraient de les lever. Cette stratégie permit aux experts de prendre la mesure du problème et de lancer une enquête internationale destinée à recueillir l'avis des autorités compétentes sur le projet de convention. La KNOB qui considérait que l'échec des mesures de 1907 tenait au défaut de signalisation des édifices protégés et à l'absence de procédure de contrôle certifiant leur démilitarisation, leur proposa de choisir un emblème pour signaler les édifices protégés puis de les inventorier dans un registre et d'instituer enfin une commission chargée de veiller au respect des 
textes qu'adopterait la communauté internationale. Le questionnaire que le comité d'experts soumit le 31 octobre 1918 au ministre des Affaires étrangères hollandais, deux mois avant l'ouverture de la conférence de la Paix, comprenait treize paragraphes: les trois premiers portaient sur les obstacles et les leviers de l'entreprise ; les neuf suivants, sur les mesures à adopter et la protection des monuments; le dernier, sur les propres lacunes du dispositif. Le mémoire explicatif qui accompagnait le document motivait sa rédaction par l'obligation morale de conserver le patrimoine en temps de guerre, faute de pouvoir éviter cette dernière. Il s'agissait d'adapter les règles de sauvegarde des biens culturels aux nouvelles stratégies militaires: les progrès techniques réalisés dans ce domaine imposaient en effet un retour sur expérience et une modification des dispositifs en vigueur. Au-delà des capacités de destruction, le comité insistait sur l'accélération des frappes, qui appelait un accroissement proportionnel de la vitesse de mobilisation des défenseurs du patrimoine. Puisqu'ils ne pouvaient s'y préparer en plein conflit, les experts hollandais en concluaient donc que la communauté devait travailler en temps de paix à la protection du patrimoine en temps de guerre. Le rapport de la KNOB parut dans une revue de droit international le 15 mai 1919, un mois avant la ratification du traité de Versailles qui donna naissance le 28 juin à la Société des Nations.

Figure 2

\begin{tabular}{|c|c|}
\hline \multicolumn{2}{|c|}{$\begin{array}{c}\text { RÉDACTEURS DES PROPOSITION DE LA NEDERLANDS OUDHEIDKUNDIGE BOND } \\
\text { SUR LA PROTECTION DU PATRIMOINE EN TEMPS DE GUERRE } \\
\text { Mai } 1918\end{array}$} \\
\hline Cuypers, J.Th. & Architecte, membre de la Commission nationale des Monuments \\
\hline Eysinga, W.J.M. van & $\begin{array}{l}\text { Professeur de droit des gens à l'Université de Leyde, délégué-adjoint des } \\
\text { Pays-Bas à la seconde Conférence de la Paix }\end{array}$ \\
\hline Gratama, S. & $\begin{array}{l}\text { Vice-Président de la Hautes-Cours des Pays Bas, Président de la } \\
\text { Commission nationale des Monuments }\end{array}$ \\
\hline Has & $\begin{array}{l}\text { daise d'Archéologie, Secrétaire de la } \\
\text { ionale des Monuments }\end{array}$ \\
\hline Overvoorde, J.C. & $\begin{array}{l}\text { Président de la } \\
\text { Section A de la }\end{array}$ \\
\hline & Professeur d'esthétique et d'histoire de l'art à l'Université d'Amsterdam \\
\hline Vollenho & Professeur à la Faculté de Droit de l'Université de Leyde \\
\hline Oordt, H.L. van & $\begin{array}{l}\text { Général Major, représentant le Ministre de la guerre et des Pays-Bas à le } \\
\text { seconde Conférence de la Paix. }\end{array}$ \\
\hline
\end{tabular}

Comité de la KNOB pour la protection des œuvres d'art et des monuments (1918).

(c) Pierre Leveau.

\section{Les réseaux de la conservation dans l'Entre-Deux- Guerres}

4 Celle-ci poursuivit-elle le travail de la KNOB ? Sur quels réseaux ses experts purent-ils s'appuyer pour tirer les leçons de la Grande Guerre? Ce ne pouvait être ni sur ceux de l'Association internationale des Académies (AIA) dissoute en 1818, ni sur ceux du Conseil international de la Recherche (IRC) qui lui succéda en 1919 : le manifeste que 93 savants allemands avaient signé en 1914 pour soutenir l'armée de Guillaume II accusée de commettre des atrocités en Belgique ${ }^{14}$ avait en effet entraîné l'éviction de l'Allemagne et de l'Autriche de ces réseaux jusqu'aux accords de Locarno en $1926^{15}$. Les défenseurs du patrimoine purent en revanche s'appuyer sur ceux de la Commission internationale de Coopération intellectuelle (CICI) créée par la Société des Nations (SDN) le 14 janvier $1922^{16}$ : ses membres n'étaient pas en effet choisis parmi les représentants d'institutions d'États 
signataires $\mathrm{du}$ Pacte, mais étaient des personnalités scientifiques indépendantes reconnues par leurs pairs, sans préjugé national ou disciplinaire. La CICI s'appuyait en France sur l'Institut international de Coopération intellectuelle (IICI) ${ }^{17}$ et dans chaque pays par des commissions nationales, finalement réunies dans un vaste réseau appelé Organisation de Coopération intellectuelle $(\mathrm{OCI})^{18}$. Elle se dota en 1926 d'une souscommission pour les Lettres et les Arts, présidée par Jules Destrée, où siégeaient notamment Paul Valéry et Henri Focillon ${ }^{19}$. C'est finalement dans ces réseaux que les savants purent faire admettre à la communauté internationale l'intérêt des questions patrimoniales ${ }^{20}$. Soutenu par J. Destrée et le gouvernement français, H. Focillon obtint en effet la création de trois nouveaux organismes : l'Office international des Musées (OIM) d'abord en $1926^{21}$, puis la Commission internationale des Arts populaires (CIAP) en $1928^{22}$, l'Office international des Instituts d'Archéologie et d'Histoire de l'Art (OIIAHA) enfin en $1932^{23}$. Les travaux de la sous-commission dans le domaine du patrimoine aboutirent en 1933 à la création d'une Commission internationale des Monuments historiques (CIMH) ${ }^{24}$ et dans le secteur des archives à celle d'un Comité international des Experts archivistes (CIEA) en $1930^{25}$. Tous ces diverticules liés à l'OCI avaient pour mission d'encourager la coopération intellectuelle pour fonder la paix sur la compréhension mutuelle, en s'appuyant sur un vaste réseau de correspondants nationaux ${ }^{26}$.

Figure 3

\begin{tabular}{|l|c|c|lr|}
\hline \multicolumn{1}{|c|}{ LES RÉSEAUX DE CONSERVATION DU PATRIMOINE } \\
Analogies structurelles
\end{tabular}

Les réseaux de conservation du patrimoine dans l'Entre-Deux-Guerres.

(c) Pierre Leveau.

5 Sur quelles questions les réseaux de conservation du patrimoine travaillèrent-ils donc dans l'Entre-Deux-Guerres ? Jusqu'à la guerre d'Espagne, l'OIM put ainsi organiser quatre conférences internationales sur les problèmes qui occupaient la communauté : d'abord à Rome en 1930 sur l'examen scientifique des œuvres ${ }^{27}$, à Athènes en 1931 sur la conservation des monuments ${ }^{28}$, à Madrid en 1934 sur la muséographie ${ }^{29}$, au Caire enfin, en 1937, sur les méthodes de fouilles ${ }^{30}$. Considérant que la conservation du patrimoine intéressait l'humanité en général et pas seulement les États ou leurs propriétaires, la SDN reconnut dès 1932 l'importance de ces questions pour la paix par une recommandation qu'elle adressa à toutes les nations, membres ou non de l'organisation, pour les inviter à coopérer et à éduquer les peuples au respect de l'héritage du passé ${ }^{31}$. Indépendamment de 
la CICI, la Fédération internationale des Associations de Bibliothécaires (IFLA) ${ }^{32}$ créée en 1929 et l'Office international de Protection de la Nature (OIPN) ${ }^{33}$ fondé en 1931 achevaient dans le secteur de la documentation et de la nature le maillage des instituts de conservation dont les réseaux avaient été coupés par la Grande Guerre. En moins de dix ans, le champ de la conservation du patrimoine, qui s'étendait des musées aux parcs naturels, en passant par les archives et les bibliothèques, les monuments et les sites, avait été arpenté et découpé. La plupart des organismes de coopération internationale qui existent aujourd'hui étaient nés (fig. $\mathbf{n}^{\circ} \mathbf{3}$ ) et les communautés scientifiques qui s'étaient formées dans ces secteurs clivés avaient adopté des doctrines différentes, mais cohérentes (fig. $\left.\mathbf{n}^{\circ} \mathbf{4}\right)$. Véritable ancêtre de l'UNESCO, l'OCI accomplit ainsi sa mission dans ce domaine.

Figure 4

\begin{tabular}{|c|c|c|}
\hline \multicolumn{3}{|c|}{$\begin{array}{l}\text { LES QUATRE CONFÉRENCES INTERNATIONALES DE L'OIM } \\
1930-1937\end{array}$} \\
\hline LIEU & OBJET & ACTES \\
\hline $\begin{array}{l}\text { Rome } \\
13-17 \text { octobre } 1930\end{array}$ & $\begin{array}{l}\text { Étude des méthodes scientifiques } \\
\text { appliquées à l'examen et à la } \\
\text { conservation des œuvres d'art. }\end{array}$ & $\begin{array}{l}\text { Manuel de la conservation et } \\
\text { de la restauration des } \\
\text { peintures, OIM-IICI, Paris, } \\
1939 .\end{array}$ \\
\hline $\begin{array}{l}\text { Athènes } \\
21-30 \text { octobre } 1931\end{array}$ & $\begin{array}{l}\text { Étude des problèmes relatifs à la } \\
\text { protection et à la conservation } \\
\text { des monuments d'art et d'histoire. }\end{array}$ & $\begin{array}{l}\text { Traité de la conservation des } \\
\text { monuments d'art et d'histoire, } \\
\text { OIM-IICI, Paris, } 1933 \text {. }\end{array}$ \\
\hline $\begin{array}{l}\text { Madrid } \\
28 \text { octobre-4 } \\
\text { novembre } 1934\end{array}$ & $\begin{array}{l}\text { Étude sur l'architecture moderne } \\
\text { des musées et les techniques de } \\
\text { muséographie. }\end{array}$ & $\begin{array}{l}\text { Traité de muséographie, } \\
\text { architecture et aménagement } \\
\text { des musées d'art, OIM-IICI, } \\
\text { Paris, 1935. }\end{array}$ \\
\hline $\begin{array}{l}\text { Le Caire } \\
9-15 \text { mars } 1937\end{array}$ & $\begin{array}{l}\text { Étude des problèmes } \\
\text { administratifs et techniques } \\
\text { concernant les fouilles. }\end{array}$ & $\begin{array}{l}\text { Manuel de la technique des } \\
\text { fouilles archéologiques, OIM- } \\
\text { IICI, Paris, } 1939 \text {. }\end{array}$ \\
\hline
\end{tabular}

Les conférences de l'OIM sur la conservation du patrimoine et sa doctrine.

(c) Pierre Leveau.

\section{L'Office international des Musées contre le pacte Roerich}

Comment expliquer, dans ces conditions, que ce réseau d'experts n'ait pas immédiatement travaillé sur la protection du patrimoine en temps de guerre ? Il leur fut impossible de rouvrir le dossier légué par la KNOB entre 1919 et 1937, même après l'échec de la conférence pour le Désarmement en 1933. Hantée par le souvenir de la Grande Guerre qui devait être la "Der des ders », la SDN bloqua systématiquement son examen, ce qui ne signifie pas qu'elle l'ait ignoré. J. Destrée qui devint président de l'OIM en 1930 avait, en effet, fait un choix différent, conforme à ses positions socialistes et pacifistes ${ }^{34}$. Considérant que la guerre prend naissance dans l'esprit des hommes et que ses germes doivent être combattus par l'éducation, il estimait que l'attachement des peuples à leur patrimoine était le plus sûr moyen de le conserver et qu'aucune loi ne serait jamais respectée si ce sentiment n'était pas d'abord cultivé. Dès 1923, il avait imposé cette doctrine à la CICI lors d'une enquête sur la recherche archéologique où se posa la question de savoir s'il valait mieux conserver in situ les découvertes faites dans les 
territoires sous mandat, au risque de voir des pilleurs se spécialiser dans leur commerce, ou au contraire autoriser leurs inventeurs à les exporter et à les conserver dans leur propre pays dans l'intérêt de l'humanité. Optant pour la première solution et tenant les territoires sous mandats pour le laboratoire d'essai d'une politique d'éducation au patrimoine, J. Destrée maintint cette position jusqu'à sa mort en 1936 : le sentiment de respect qu'inspire aux générations présentes le legs hérité du passé était selon lui le vrai remède aux exactions dont les pays occupés avaient été victimes durant la Grande Guerre ${ }^{35}$. Pour éviter le fléau du pillage et du vandalisme, il fallait non seulement savoir conserver le patrimoine, mais aussi constituer un réseau d'échanges international pour communiquer le sentiment de respect que chaque nation éprouve pour le sien à toutes les autres et élever ainsi les peuples à l'idée d'un patrimoine commun de l'humanité, au-delà des frontières et des égoïsmes nationaux ${ }^{36}$. Selon les membres de l'OIM, internationalistes convaincus, c'était la première leçon à tirer de la Grande Guerre. C'est sur les modalités de ces échanges que porta finalement la dernière conférence qu'ils organisèrent au Caire. Qui veut la paix éduque les peuples aurait pu être la devise de ces humanistes, qui pensaient que les échanges culturels favorisent leur compréhension mutuelle.

7 Tous les autres pays avaient-ils tiré la même leçon du conflit? Sans doute pas et ce n'est pas en Europe, mais aux États-Unis, sous l'égide de l'Union Panaméricaine (UP), que la question de la protection du patrimoine en temps de guerre resurgit. L'UP et la SDN signèrent en 1928 le pacte Briand-Kellogg qui condamnait le recours à la guerre dans le règlement des conflits. Ce Pacte inspira au peintre russe Nicolas Roerich l'idée de reprendre le $\mathrm{VI}^{\mathrm{e}}$ vœu du congrès de 1889 et d'inventer un symbole pour combler la lacune du $27^{\mathrm{e}}$ article de la IV ${ }^{\mathrm{e}}$ convention de 1907, suivant sans les nommer F. Vetter et la KNOB. Émigré à New York, le théosophe et ancien directeur de la Société impériale d'Encouragement des Arts de Saint-Pétersbourg proposait une nouvelle fois de créer une bannière qui rendrait aux biens culturels le même service que la Croix-Rouge aux hommes. Les institutions consacrées à la science et l'éducation devaient selon lui être sanctuarisées à l'instar des hôpitaux: un drapeau blanc pour la paix, orné d'un cercle rouge symbolisant la culture enfermant trois sphères représentant la religion, la science et l'art, désignerait les édifices auxquels la communauté internationale aurait accordé ce statut par une convention consacrant leur inviolabilité. Disposant d'un solide réseau, le théosophe confia à l'attaché juridique de son musée, le français Georges Chklaver, maître de conférences à l'Institut des Hautes Études internationales de la faculté de Paris, le soin de rédiger le projet de convention qui protégerait les monuments. Le premier article du nouveau pacte qu'il proposait à la SDN et à l'UP stipulait que les biens culturels et les institutions chargées de les conserver seraient considérés comme neutres. Effaçant les frontières qui existaient entre les sciences, les arts et l'éducation, le deuxième englobait sous l'idée de "monuments de l'esprit» toutes les œuvres protégées. Le troisième décrivait le symbole protecteur des trois secteurs réunis sous une même bannière et le quatrième expliquait comment le Pacte serait mis en œuvre: ses signataires transmettraient la liste des sites protégés à une organisation internationale qui la communiquerait aux belligérants pour qu'ils les épargnent. Le cinquième article précisait que leur usage à des fins militaires entraînerait leur radiation et le dernier autorisait les signataires du Pacte à le dénoncer à tout moment.

8 Comment l'OIM accueillit-il ce Pacte qui tirait comme lui les leçons de la Grande Guerre? En juin 1929, N. Roerich et G. Chklaver fondèrent à Paris l'Association française des Amis du Roerich Museum, qui devait relayer leur entreprise sur le Vieux Continent. Cette base 
leur permit l'année suivante de commencer à rallier les Européens à leur cause avant de soumettre leur projet à la SDN et à l'UP dans le cadre du pacte Kellogg-Briand. Le 14 septembre 1931, ils organisèrent à Bruges un premier congrès pour fédérer les associations de défense du patrimoine autour du texte que $\mathrm{G}$. Chklaver avait rédigé ${ }^{37}$. Ce congrès fut suivi d'un autre l'année suivante où fut créé la fondation Roerich pour la Paix, l'Art, les Sciences et le Travail, chargée de gérer les dons des mécènes et promouvoir les idées du théosophe. En 1933, l'association réunissait de nombreux directeurs de musées et de grands noms de l'aristocratie européenne qui soutenaient l'entreprise dans l'espoir de voir un jour flotter sur leurs belles demeures la bannière de la culture et la paix qui devait les protéger des horreurs de la guerre ${ }^{38}$. Si la fondation Roerich se prévalait à l'époque du soutien de l'OIM, il n'en était rien en réalité. Soucieux d'encourager les bonnes volontés, son président J. Destrée avait, il est vrai, accueilli favorablement son projet: dans un article publié le 31 mai 1930, il salua l'initiative de $\mathrm{N}$. Roerich et $\mathrm{G}$. Chklaver, tout en rappelant que leurs propositions n'ajoutaient rien aux dispositions que la communauté internationale avait adoptées depuis $1907^{39}$. S'il suggérait d'associer au projet l'IICI, en confiant à l'Institut parisien la mission de communiquer la liste des monuments protégés conformément au quatrième article du Pacte, le ton diplomatique de J. Destrée cachait en réalité un avis bien plus tranché. En juillet 1930, la CICI rejeta en effet le pacte Roerich en retenant les arguments qu'exposa le secrétaire général de l'OIM, E. Foundoukidis ${ }^{40}$. Juridiquement, le texte de G. Chklaver accréditait l'idée absurde que l'on puisse faire la guerre en respectant l'œuvre de l'Esprit et contredisait selon lui le pacte Kellogg-Briand qui voulait la mettre hors la loi. Techniquement, il pouvait encourager les belligérants à utiliser des armes chimiques, contre la convention de 1925 qui les interdisaient, ce type d'armement permettant de tuer l'ennemi sans endommager les bâtiments. Moralement, il renversait aussi l'échelle des valeurs humaines, en accordant plus d'importance aux biens culturels qu'aux individus mêmes. En donnant une bannière aux défenseurs des monuments, il divertissait enfin l'opinion et détournait les bonnes volontés de l'action concrète. Il participait donc au nihilisme ambiant selon E. Foundoukidis, qui estimait que l'OIM ne pouvait cautionner une entreprise aussi inutile que dangereuse et contre-productive. La CICI adopta cette position lors de sa session de juillet 1931, où son président Gilbert Murray réduisit à l'absurde le pacte Roerich par un dernier argument: puisque celui qui aurait la sagesse d'épargner les œuvres d'art en temps de guerre aurait aussi celle de ne pas la faire, le texte était finalement sans objet ni destinataire. C'est pourquoi la question de la protection des monuments en cas de conflit fut finalement rayée du programme de la conférence d'Athènes qui eut lieu l'année même.

Quelles étaient donc les positions des défenseurs des monuments lors de la conférence pour le Désarmement en 1932 ? Ils se divisaient en deux camps : celui des partisans du pacte Roerich qui pensaient qu'il fallait une loi pour protéger le patrimoine culturel de la guerre et de la barbarie, celui des membres de la CICI dont le projet venait d'être consacré par une recommandation de la SDN affirmant qu'il fallait sensibiliser les peuples à son respect (fig. $\mathbf{n}^{\circ} 5$ ). Entre la loi et le sentiment, un océan séparait ces deux camps dont les frontières s'alignèrent finalement sur celles de la SDN et de l'UP, lorsque cette dernière adopta le pacte du théosophe, tandis que la première s'en tenait aux conventions passées à La Haye. Alors que l'Allemagne nazie quitta la conférence sur le Désarmement en même temps que la SDN le 14 octobre 1933, les Amis du Roerich Museum organisèrent le 17 novembre à Washington leur troisième congrès, rebaptisé convention internationale 
pour la Paix. Le 6 décembre, la CICI faisait officiellement connaître son refus d'adhérer au pacte Roerich, tandis que la VII ${ }^{\mathrm{e}}$ conférence de l'UP réunie à Montevideo recommandait son adoption ${ }^{41}$. Le 15 avril 1935, les États-Unis et vingt autres pays d'Amérique latine ratifièrent ainsi le Pacte alors que des bataillons de soldats emmenés par des généraux férus d'humanités allaient bientôt montrer aux experts de la SDN que la frontière qui séparait la civilisation de la barbarie ne passait pas où ils croyaient.

Figure 5

\begin{tabular}{|c|c|}
\hline \multicolumn{2}{|c|}{$\begin{array}{l}\text { LE CLIVAGE DES DÉFENSEURS DU PATRIMOINE DANS L'ENTRE-DEUX-GUERRE } \\
1929-1935\end{array}$} \\
\hline DATE & ÉVÉNEMENT \\
\hline 17 juin 1925 & rotocole de Genève prohibant l'emploi de gaz toxiques. \\
\hline 27 août 1928 & Signature du Pacte acte Brian-Kellogue pour la Paix. \\
\hline Jui & $\begin{array}{l}\text { Rédaction du Pacte Roerich et création de l'Association française } \\
\text { des amis du Roerich Museum. }\end{array}$ \\
\hline $31 n$ & Réponse de J. Destrée aux rédacteurs du Pacte Roerich. \\
\hline re 1931 & $\mathrm{~s}$ des Amis du Roerich Mus \\
\hline Juillet 1931 & u Pacte Roerich par la $\mathrm{ClCl}$ \\
\hline 7 septembre 19 & $2^{\mathrm{e}}$ congrès des Amis du Roerich Mus \\
\hline 2 février 1932 & Conférence pour la réduction des armements à Genève \\
\hline 14 octobre 1933 & L'Allemagne quitte la conférence pour le désarmement et la SDN. \\
\hline 17 novembre 1933 & $3^{\mathrm{e}}$ Congrès des Amis du Roerich Museum à Washington \\
\hline 6 décembre 1933 & $\mathrm{La} \mathrm{CICl}$ réitère son refus du Pacte Roerich \\
\hline 27 décembre 1933 & La VII ${ }^{\mathrm{e}}$ conférence de l'UP recommande l'adoption du Pacte Roerich. \\
\hline 1 & 25 États membres de l'UP ratifient le Pacte \\
\hline
\end{tabular}

Chronique du différend de la SDN et de l'UP sur la protection du patrimoine en temps de guerre. (c) Pierre Leveau.

\section{La guerre civile espagnole et le retour du refoulé}

La montée en puissance des nationalismes contraignit-elle l'OIM à réviser ses positions en 1933 ? Il avait déjà tiré deux leçons de la Grande Guerre: l'une sur la nécessité de constituer un réseau mondial d'experts partageant les mêmes valeurs, l'autre sur l'intérêt de l'utiliser pour promouvoir l'idée d'un patrimoine commun de l'humanité qui devait servir la paix. Mais la guerre civile espagnole qui éclata le 17 juillet 1936 le contraignit finalement à reprendre l'enquête lancée par la KNOB dix-huit ans plus tôt. Constatant l'absence de délégation espagnole au XIV ${ }^{e}$ congrès international d'Histoire de l'Art (CIHA) réuni à Bâle le 9 septembre, ses membres avaient demandé à l'OIM de s'assurer que le Trésor d'Espagne serait protégé et que les établissements chargés de le conserver poursuivraient leur mission durant le conflit ${ }^{42}$. La France, l'Angleterre et l'Italie avaient institué le même jour à Londres un comité international de Non-Intervention, interdisant à leur gouvernement la livraison d'armes aux belligérants. Constatant ce blocage, la Bolivie saisit le 7 octobre la SDN de la question de l'intervention dans le conflit espagnol et déclencha une procédure consultative de tous ses diverticules, dont l'OCI. Celle-ci demanda immédiatement au comité de direction de l'olM de tenir une réunion extraordinaire, qui ouvrit ainsi les portes de la CICI à l'enquête de la KNOB refoulée jusqu'alors en même temps qu'au vœu du CIHA. Le 12 octobre, l'OIM put ainsi faire connaître sa stratégie, consistant à consulter un expert, Charles de Visscher, membre de l'Académie de Droit international à La Haye, puis à remettre un rapport à la CIC où seraient rappelées les résolutions déjà prises ${ }^{43}$, tout en publiant dans la revue Mouseion 
toutes les pièces du dossier accompagnées d'autres articles sur le sujet. Il s'agissait de rouvrir le dossier constitué en 1931 lors des discussions sur le pacte Roerich, sans changer les positions adoptées alors par l'organisation qui faisait de l'éducation et de la coopération intellectuelle le nerf de la guerre en matière de conservation. Voué à la neutralité et paralysé par ses statuts, l'OIM reconnaissait par ailleurs son impuissance : la SDN pouvait intervenir dans des différends entre États, mais pas en cas de guerres civiles, ce qui plaçait l'organisation devant un vide juridique où elle allait sombrer. Après le pacte Roerich, le conflit espagnol ouvrit en 1936 un second front dans le combat pour la protection du patrimoine, où l'OIM acheva de tirer les leçons de la Grande Guerre.

11 Mais ces leçons furent-elles seulement comprises par les gouvernements auxquels elles s'adressaient ? L'épisode du sauvetage du Trésor artistique d'Espagne met en évidence les limites de l'entreprise. Lorsque le musée du Prado ferma ses portes le 30 août 1936 après les premiers bombardements de Madrid ${ }^{44}$, le conservateur français Raymond Escholier n'imaginait pas que la communauté internationale puisse assister à sa destruction sans intervenir et, le 11 septembre, appela à constituer des brigades internationales de défense des œuvres d'art, à mi-chemin entre celles du Komintern et les Juntas Espagnoles ${ }^{45}$. S'indignant de la passivité de l'OIM, il espérait sauver les collections du Prado en réalisant au Louvre ce qu'il avait réussi un an plus tôt au Petit Palais. Le rapprochement de la France et de l'Italie entamé après les accords de Locarno en 1926, puis scellé par le Pacte à Quatre de juin 1933, lui avait permis d'obtenir sur ordre de Mussolini 280 tableaux pour son exposition sur l'art italien de Cimabue à Tiepolo ${ }^{46}$, alors qu'il en avait demandé 180 . Le Duce demandait de surcroît au sénateur Borletti de donner à la France une partie de sa collection contemporaine exposée au Jeu de Paume, en remerciement des accords passés avec Pierre Laval sur la Tunisie et l'Éthiopie ${ }^{47}$. Tandis que l'exposition du Petit Palais témoignait des vertus passées de l'Italie, celle du Jeu de Paume leur promettait un avenir fasciste. Loin d'être désintéressée, l'opération permettait ainsi à Mussolini de reprendre à son compte, aux yeux du monde, l'héritage artistique du pays. Très loin de l'idée d'un patrimoine commun de l'humanité que l'oIM promouvait en tirant les leçons de la Grande Guerre, l'exposition de 1935 prolongeait une tradition historiographique héritée du XIX ${ }^{\mathrm{e}}$ qui voyait dans l'art l'expression du génie des peuples et qui servait finalement le projet politique des gouvernements nationalistes ${ }^{48}$. La même tragédie culturelle se rejoua quatre ans plus tard en Suisse. Il existait dès 1936 deux gouvernements en Espagne, nationaliste à Burgos, républicain à Madrid, pour lesquels le patrimoine était un enjeu politiquement fort, puisque cet attribut symbolique leur assurerait la reconnaissance du peuple ${ }^{49}$. L'OIM assista impuissant à leur lutte pour cet héritage jusqu'à la Seconde Guerre mondiale ${ }^{50}$. Reprenant le projet de R. Escholier, l'administration française pensa, d'abord, à organiser au Louvre une exposition en hommage au peuple espagnol, pour mettre les chefs-d'œuvre du Prado à l'abri des bombes. Mais tandis que les négociations entre la France et la République d'Espagne semblaient près d'aboutir en mai 193751, le projet périclita lors de l'Exposition internationale de Paris où Picasso présenta Guernica: le gouvernement de Burgos opposa un veto auquel la France dut se plier. L'échec des négociations obligea les défenseurs du patrimoine à élaborer de nouvelles stratégies d'exfiltration des œuvres que menaçaient les bombardements et l'avancée des troupes nationalistes. Le 12 octobre 1937, José Renau, directeur général des Beaux-Arts de la République espagnole, appela $\mathrm{E}$. Foundoukidis à former un comité international de sauvegarde, par un argument qui tranchait avec ceux des nationalistes et qui s'appuyait directement sur l'idée d'un patrimoine commun de l'humanité : il fallait selon lui sauver le Trésor d'Espagne, non 
seulement dans l'intérêt du peuple espagnol qui en était dépositaire, mais dans celui de l'humanité en général, parce qu'il témoignait du génie des hommes, au-delà de celui d'une nation ${ }^{52}$

L'OIM parvint-il dans ces conditions à défendre le patrimoine mieux qu'on ne le fit avantguerre? La proposition que l'organisation fit en novembre 1937 d'instituer une Organisation internationale de Sauvegarde ${ }^{53}$ en réponse à la demande de J. Renau n'aboutit pas plus que les tentatives de la SDN pour régler le conflit des Nationalistes et des Républicains. Le projet fut abandonné au profit d'un comité international pour la Sauvegarde des Trésors d'Art espagnol (CISTAE) finalement créé le 29 janvier 1939 pour transporter à Genève le patrimoine conservé par les Républicains bloqués à Figueras ${ }^{54}$. Présidé par le mécène D. David-Weill et fondé par José Maria Sert et Joan i Estelrich Artigues, respectivement peintre et député catalan, il réunissait les directeurs de la plupart des grands musées d'Europe, tous membres de l'OIM (fig. $\mathbf{n}^{\circ} \mathbf{6}$ ), l'organisation étant simultanément exclue de l'opération. Le 3 février, le gouvernement républicain fut moralement contraint de signer à Figueras la convention par laquelle il confiait au CISTAE la mission de remettre le Trésor artistique d'Espagne au secrétariat de la SDN ${ }^{55}$. Le premier article de cette convention indiquait que les Républicains étaient les légataires du bien et le deuxième désignait le secrétaire de la SDN comme son dépositaire ; les cinq suivants déléguaient son transport et son inventaire aux experts du CISTAE, tandis que les deux derniers stipulaient enfin qu'il était la propriété commune de la nation espagnole et qu'il serait intégralement restitué à son gouvernement lorsque le pays connaîtrait à nouveau la paix, le président de la JCIPTA rappelant dans une note son inaliénabilité absolue. L'affaire se dénoua enfin le 14 février lorsque le train contenant le Trésor artistique d'Espagne fut déchargé en gare de Genève ${ }^{56}$. Le Conseil fédéral suisse reconnut le jour même le gouvernement de $\mathrm{F}$. Franco, l'autorisant ainsi à réclamer ces biens, conformément au dernier article de la convention de Figueras. La SDN le lui restitua le 30 mars et le dictateur hérita ainsi du droit de conserver le patrimoine artistique espagnol que les Républicains avaient sauvé dans l'intérêt de l'humanité et pas seulement dans celui du peuple espagnol ${ }^{57}$. L'Espagne quittait la SDN le 8 mai, si bien que l'exposition des chefs-d'œuvre du Prado par laquelle le CISTAE espérait se rembourser du sauvetage ne put avoir lieu au palais des Nations ni être patronnée par la SDN. Elle fut inaugurée le $1^{\mathrm{er}}$ juin au musée d'Art et d'Histoire de la ville de Genève, sans qu'aucun membre du CISTAE, de l'OIM et de la SDN y soit invités. Sur le plan symbolique, elle attribuait les œuvres exposées au génie espagnol plutôt qu'à celui de l'humanité. Elle portait l'organisation de la manifestation au crédit du gouvernement de F. Franco, plutôt qu'à l'esprit de la République et à la coopération intellectuelle. Comme celle « De Cimabue à Tiepolo » quatre ans plus tôt, elle opéra ainsi un recyclage nationaliste de biens que les Républicains et l'OIM tenaient pour un patrimoine commun de l'humanité. La conservation du Trésor d'Espagne redevint une affaire nationale après que le gouvernement de Franço eût encaissé à Genève le bénéfice symbolique de son sauvetage ${ }^{59}$. 


\begin{tabular}{|l|l|c|}
\hline \multicolumn{3}{|c|}{ COMITÉ INTERNATIONAL } \\
POUR LA SAUVEGARDE DES TRÉSORS ARTISTIQUES ESPAGNOLES \\
Janvier 1939
\end{tabular}

Le Comité international pour la Sauvegarde des Trésors artistiques espagnols (1939).

(c) Pierre Leveau.

\section{La leçon de l'histoire : l'idée de patrimoine commun de l'humanité}

13 Quelles leçons l'OIM tira-t-il de la guerre d'Espagne ? Elle l'obligea à revenir sur celles de la Grande Guerre, censurées jusqu'alors : il republia dans le numéro de décembre 1937 de la revue Mouseion l'enquête menée par la KNOB sur la protection du patrimoine, suivie du rapport que C. de Visscher remit à la CICI en 1936 et de l'appel que J. Renau avait lancé à la communauté internationale. En septembre 1938, l'Assemblée de la SDN approuva finalement l'avant-projet de convention internationale sur la Protection des Monuments et des Euvres que le Comité réuni par C. de Visscher en novembre 1937 lui soumit, poursuivant le projet de la KNOB qui avait eu raison trop tôt $\left(\right.$ fig. $\mathbf{n}^{\circ} 7$ ). Le texte comprenait treize articles, suivis d'un règlement exécutoire. Réaliste, son préambule affirmait enfin que la réflexion en temps de paix devait être consacrée à la sauvegarde du patrimoine en temps de guerre ${ }^{60}$. Il levait ainsi l'obstacle du pacifisme, à la suite du pacte Roerich. Mais à la différence de ce dernier, il décrivait surtout le dispositif qui permettrait à la nouvelle convention de s'appliquer, corrigeant ainsi le défaut que la KNOB avait déjà pointé dans celle de La Haye. Ses trois premiers articles rappelaient l'importance du principe de coopération intellectuelle dans la conservation et définissaient les devoirs qui incomberaient aux signataires, à savoir l'anticipation, l'information, la sensibilisation et l'assistance mutuelle; le troisième insistait plus particulièrement sur la nécessité de sensibiliser les troupes au respect du patrimoine en général, l'éveil de ce sentiment conditionnant leur obéissance à la loi selon la $\mathrm{CICI}$; les suivants précisaient les conditions que les monuments et les dépôts devaient satisfaire 
pour bénéficier d'une protection: être éloignés d'objectifs stratégiques, être démilitarisés, pouvoir enfin être inspectés et être signalés. L'article huit condamnait en particulier le vandalisme et les deux suivants légalisaient l'entraide des États. Les derniers et le règlement exécutoire prévoyaient enfin qu'une conférence générale soit instituée pour veiller au respect de ces clauses et qu'une commission internationale d'inspection soit créée pour le vérifier. Les édifices qui rempliraient ces conditions seraient inscrits dans un registre en temps de paix et signalés par un emblème en temps de guerre, consistant, selon l'article sept, en un triangle bleu clair inscrit dans un disque blanc. Accompagné d'un long commentaire d'E. Foundoukidis, le texte fut publié dans l'avant-dernière livraison de Mouseion en $1939^{61}$. Les différents chapitres de ce manuel de protection du patrimoine en temps de guerre portaient sur la prévention des risques, le contrôle de l'environnement, l'emballage, le transport et le stockage des œuvres. Ce volume achevait ainsi tragiquement le paradigme que l'OIM avait construit à Rome, à Athènes, à Madrid puis au Caire. Tirant enfin la troisième et dernière leçon de la Grande Guerre, son secrétaire général y dénonçait le mal qui avait fait échouer l'entreprise, à savoir le nationalisme qui réduisait l'histoire de l'art à exalter le génie des peuples. Il indiquait le remède que l'OIM avait voulu lui apporter : le comparatisme que véhicule l'anthropologie culturelle et qu'encourage le libre échange entre les musées ${ }^{62}$. Internationaliste, il concluait que l'histoire imposait désormais aux historiens d'art de changer de paradigme et d'adopter celui que l'organisation promouvait depuis sa création. Philosophiquement, l'obligation morale de respecter le patrimoine de l'ennemi en temps de guerre devait se fonder sur l'idée que les nations n'étaient pas les propriétaires mais les dépositaires d'un héritage commun. Scientifiquement, il fallait donc leur montrer que les chefs-d'œuvre de l'art n'étaient pas la simple expression du génie des peuples, mais le produit des échanges qui fécondaient les talents aux carrefours des cultures, c'est-à-dire de la coopération intellectuelle. À l'aube de la Seconde Guerre mondiale, le secrétaire général de l'OIM expliquait ainsi que ce patrimoine commun était celui de l'humanité et qu'il ne pourrait être protégé en temps de guerre que lorsque les nations en auraient admis l'idée. On ne pouvait en effet demander aux belligérants de le respecter comme si c'était le leur qu'en séparant les concepts de patrimoine et de nation, leur union justifiant à l'inverse son instrumentalisation politique. 


\begin{tabular}{|c|c|c|}
\hline DE LA CONVENT & $\begin{array}{l}\text { PREMIER COMITÉ DE RÉDACTION } \\
\text { UR LA PROTECTION DU PATRIMOINE EN TEMI } \\
\text { Novembre } 1937\end{array}$ & E GL \\
\hline MON & TITRE & RÉS. \\
\hline & Secrétaire général & \\
\hline Foundoukidis, E. & $\begin{array}{l}\text { Secrétaire général de l'OIM, Chef du Département d'Art, } \\
\text { d'Archéologie et d'Ethnologie à l'IICI }\end{array}$ & Paris \\
\hline & Experts & \\
\hline La Pradelle, G. de & Professeur de Droit international à la Faculté de Paris & Paris \\
\hline Maclagan, E. & Directeur du Victoria and Albert Museum & Londres \\
\hline Madariaga, S. de & Président du comité de direction de l'OIM & Londres \\
\hline $\begin{array}{l}\text { Commandant } \\
\text { Moineville }\end{array}$ & $\begin{array}{l}\text { Inspecteur de la défense anti-aérienne auprès du } \\
\text { Ministère de l'Air }\end{array}$ & Paris \\
\hline Pellati, F. & Inspecteur supérieur des Beaux-Arts & Rome \\
\hline S.E. Politis & Ministre de la Grèce à Paris & Paris \\
\hline Capitaine Sas & Etat Major néerlandais & La Haye \\
\hline Schmidt-Degner, F. & Directeur général du Rijksmuseum & Amsterdam \\
\hline Stix, A. & Premier Directeur du Kunsthistorische Museum & Vienne \\
\hline Verne, $\mathrm{H}$. & Membre de l'Institut, Directeur des Musées nationaux & Paris \\
\hline Visscher, Ch. de & Juge à la Cours permanente de Justice internationale & La Haye \\
\hline
\end{tabular}

Premier comité de l'OIM pour la Protection du patrimoine en temps de guerre (1939).

(c) Pierre Leveau.

14 L'OIM parvint-il à corriger le bégaiement de l'histoire ? Ses efforts permirent en tout cas aux responsables du patrimoine d'être prêts lorsque la Seconde Guerre éclata. La SDN chargea, à la veille du conflit, le gouvernement néerlandais de transmettre l'avant-projet sur la protection du patrimoine qu'elle avait approuvé en septembre 1938 et l'IICI de réunir un nouveau comité d'experts pour intégrer les amendements qui seraient proposés (fig. n8). Il se réunit d'abord à Paris le 22 décembre 1938, puis à La Haye le 6 février 1939. Si parmi les États consultés, quatre seulement proposèrent des amendements, les deux tiers se dirent prêts à se rendre à la conférence diplomatique que la SDN organiserait ${ }^{63}$, tandis que les autres se ralliaient simplement aux principes énoncés ${ }^{64}$. L'Angleterre, la Suisse et les États-Unis jugeaient à l'inverse la proposition irréaliste compte tenu des circonstances ; l'Allemagne et l'Italie accusaient une fin de non-recevoir et seul le Japon refusa explicitement le texte. Mais la conférence diplomatique au cours de laquelle les conventions sur la protection du patrimoine auraient dû être ratifiées n'eut jamais lieu: le $1^{\text {er }}$ septembre 1939, l'Allemagne nazie envahit la Pologne et trancha tragiquement le dilemme que R. Musil formulerait plus tard en égalisant et en niant la valeur des vies et des monuments. On ne pouvait espérer que les inventeurs de la guerre totale respectent le patrimoine. Redoutant un nouveau Guernica, le président des États-Unis lança le même jour un appel contre les bombardements des populations civiles ${ }^{65}$, auquel les gouvernements du Royaume-Uni et de la France répondirent en s'engageant à limiter leurs frappes aux objectifs militaires. Cynique, Adolf Hitler approuva immédiatement cette déclaration, alors que le monde basculait dans la guerre. Le 3 septembre, tous s'engagèrent ainsi à épargner les témoignages de la civilisation au même titre que les populations civiles et l'OIM salua cette promesse par un communiqué exposant les grandes lignes de la convention sur laquelle son comité de juristes travaillait encore. Le 8 septembre, l'organisation appela enfin les responsables des monuments, des bibliothèques et des musées à protéger leurs trésors artistiques par un texte radiodiffusé en français puis en anglais rappelant le principe selon lequel la conservation du patrimoine intéressait la communauté des peuples ${ }^{66}$. Dans le même temps, les pays 
frontaliers de l'Allemagne décrochaient leurs collections ${ }^{67}$. C'est dans ce contexte que E. Foundoukidis clôt finalement l'enquête de l'OIM sur la protection du patrimoine en publiant toutes les pièces du dossier dans Mouseion et en demandant à ses lecteurs d'attendre la fin du conflit pour le rouvrir6s. C'est sur ce legs que s'acheva en décembre 1939 l'histoire des réseaux de conservation dans l'Entre-Deux-Guerres et que commença celle des défenseurs du patrimoine qui poursuivirent leur œuvre.

Figure 8

\begin{tabular}{|c|c|c|}
\hline \multicolumn{3}{|c|}{$\begin{array}{l}\text { SECOND COMITÉ DE RÉDACTION } \\
\text { DE LA CONVENTION SUR LA PROTECTION DU PATRIMOINE EN TEMPS DE GUERRE } \\
\text { Décembre } 1938\end{array}$} \\
\hline MON & TITRE & RÉS. \\
\hline \multicolumn{3}{|c|}{ Secrétaire général } \\
\hline Foundoukidis, E. & $\begin{array}{l}\text { Secrétaire général de l'OIM, Chef du Département d'Art, } \\
\text { d'Archéologie et d'Ethnologie à l'IICI }\end{array}$ & Paris \\
\hline \multicolumn{3}{|c|}{ Experts } \\
\hline Visscher, Ch. de & Juge à la Cour Permanente de Justice internationale & La Haye \\
\hline Kosters, J. & Vice-Président à la Cour suprême des Pays-Bas & La Haye \\
\hline Basdevant, J. & $\begin{array}{l}\text { Professeur à la Faculté de Droit de I'Université de Paris, } \\
\text { Conseiller juridique au Quai d'Orsay }\end{array}$ & Paris \\
\hline M. Picard, M. & Professeur à la Faculté de Droit de l'Université de Paris, & Paris \\
\hline Gutteridge, H.C. & Professeur à la Faculté de Droit de Cambridge & Cambridge \\
\hline Weiss, R. & Conseillé juridique de l'IICl & Paris \\
\hline Schmidt-Degner, F. & Directeur général du Rijksmuseum & Amsterdam \\
\hline
\end{tabular}

Second comité de l'OIM pour la Protection du patrimoine en temps de guerre (1939).

(c) Pierre Leveau.

\section{Conclusion}

Quelles leçons les défenseurs du patrimoine ont-ils donc tirées de la Grande Guerre? Retenons-en au moins quatre: premièrement, la nécessité de constituer un réseau mondial de conservation, favorisant la coopération intellectuelle et les échanges entre les peuples, deuxièmement, l'obligation d'utiliser ce réseau pour promouvoir l'idée d'un patrimoine commun de l'humanité, garantissant la paix par la compréhension mutuelle, troisièmement, l'intérêt de changer de paradigme pour étudier les échanges culturels entre les peuples, au lieu de célébrer le génie national, la nécessité, enfin, de fonder la protection du patrimoine sur un sentiment - le respect des biens du passé - avant de le fonder sur la loi. Aux partisans du pacte Roerich, qui pensaient qu'il suffisait de légiférer pour protéger les monuments, les experts de l'oIM objectèrent que les conventions ratifiées en temps de paix ne seraient jamais suivies en temps de guerre si les peuples n'étaient pas d'abord sensibilisés à l'idée d'un patrimoine commun de l'humanité. Aux gouvernements nationalistes qui instrumentalisaient leur héritage à des fins politiques, ils rappelèrent qu'ils devaient moins se considérer comme les propriétaires de ces biens que comme leurs dépositaires. La Grande Guerre justifiait selon eux ces conclusions et son souvenir, qui les hantait, explique les pratiques discursives qu'ils adoptèrent, marquées par l'internationalisme et le pacifisme. La Seconde Guerre mondiale montra cependant les limites de leur modèle et conduisit, comme on le sait, à la ratification en 1954 d'une « convention pour la Protection des biens culturels en cas de conflit armé »"${ }^{69}$, puis en 1996 à la création du comité international du Bouclier bleu ${ }^{70}$, dont on a rappelé la préhistoire. Après plus d'un siècle de traduction, c'est-à-dire de transmissions doublées de 
négociations, on peut en conclure que le testament de l'oIM et le vœu du congrès de 1889 ont bien été exécutés.

\section{NOTES}

1. - LEVEAU, Pierre. "Problème de l'apolitique de la conservation-restauration ». ConservationRestauration des Biens Culturels, 2011, n²9, p. 5-26, Fig. 2 pour l'inventaire complet de ces archives.

2. - LÉON, Paul. Art et artistes aujourd'hui. Paris : Fasquelle, 1926, p. 9-30.

3. - LENIAUD, Jean-Michel. Les archipels du passé. Paris : Fayard, 2002, p. 245-249.

4. - RIEGL, Aloïs. Der moderne DenkmalKultus, sein Wesen, sein Entstehung. Wien : W. Braumüller, 1903.

5. - DVORAK, Max. Katechismus der Denkmalpflege. Wien : J. Bard, 1916-1918.

6. - BLOWER, Jonathan. "Max Dvorak and Austrian Denkmalpflege at War ». Journal of Art Historiography, 2009, $\mathrm{n}^{\circ} 1$, p. 1-19.

7. - MUSIL, Robert. L'homme sans qualité. Paris : Seuil, 1957, t. 2, § 89, p. 116.

8. - NORMAND, Charles. Congrès international pour la Protection des CEuvres d'Art et des Monuments, tenu à Paris du 24 au 29 juin 1889. Paris : Imprimerie nationale, 1889.

9. - Convention (IV) concernant les lois et coutumes de la guerre sur terre et son Annexe: Règlement concernant les lois et coutumes de la guerre sur terre. La Haye, 18 octobre 1907.

10. - Convention (IX) concernant le bombardement par les forces navales en temps de guerre. La Haye, 18 octobre 1907.

11. - GERMANN, Georg. « Henry de Geymüller, un expert polyglotte et cosmopolite ». Dans RECH, Roland. Victor Hugo et le débat patrimonial. Paris : INP/Somogy, 2003, p. 103-120.

12. - VETTER, Ferdinand. «Friede dem Kunstwerk! Zwischenstaatliche Sicherung der Kunstdenkmäler im Kriege als Weg zum künftigen dauerhaften Frieden». Denkschrift über die Grundlagen eines dauerhaften Friedens-Vertrages / herausgegeben vom Schweizerischen Komitee zum Studium der Grundlagen eines dauerhaften Friedensvertrages. Olten : W. Trösch, 1917.

13. - « La protection des Monuments et objets historiques et artistiques contre les destructions de la guerre - Proposition de la Société Néerlandaise d'Archéologie ». Mouseion, n³9/40, 1937, p. 81-88. La KNOB fut fondée le 7 janvier 1899 et poursuit ses activités : http://www.knob.nl. Tous les numéros de Mouseion sont consultables sur Gallica : http://gallica.bnf.fr.

14. - «An die Kulturwelt - Ein Aufruf», Berliner Tageblatt, 4 octobre 1914 / "Appel des intellectuels allemands aux nations civilisées ». La Revue Scientifique, 14 novembre 1914.

15. - RASMUSSEN, Anne. «Réparer, réconcilier, oublier : enjeu et mythes de la démobilisation scientifique, 1919-1925». Politique, culture, société, n³, novembre-décembre 2007.

16. - RENOLIET, Jean-Jacques. L'Unesco oubliée. La Société des Nations et la coopération intellectuelle (1919-1946). Paris : Publications de la Sorbonne, 1999.

17. - MAYOUX, Jean-Jacques. L'Institut International de Coopération Intellectuelle, 1925-1946. Paris : IICI, 1947.

18. - RENOLIET, Jean-Jacques. «L'Unesco oubliée. L'Organisation de coopération intellectuelle (1919-1946) ». Dans BOEL, Jens. 60 ans d'histoire de l'Unesco. Actes du colloque international. Paris, 16-18 novembre 2005. Paris : UNESCO, 2007, p. 61-66.

19. - IICI. Cahiers des Relations artistiques. I. La Coopération Intellectuelle et les Beaux-Arts. Paris : PUF, 1927. 
20. - SDN-CICI. Commission de Coopération intellectuelle. Procès-Verbaux de session, 1922-1939. Genève : SDN, 1939.

21. - «L'ÆEuvre de coopération intellectuelle et l'Office international des Musées ». Mouseion, $\mathrm{n}^{\circ} 1$, avril 1927, p. 3-16.

22. - ROGAN, Bjarne. «Folk Art and Politics in Inter-War Europe : An Early Debate on Applied Ethnology ». Folk life, $n^{\circ} 45$, p. 7-23.

23. - «Office des Instituts d'Archéologie et d'Histoire de l'Art». Bulletin de la Coopération Intellectuelle, $\mathrm{n}^{\circ} 57 / 58,1935, \mathrm{p} .454$.

24. - « L'activité de l'Office international des musées ». Mouseion, $n^{\circ} 23 / 24,1933$, p. 243-244.

25. - «Comité d'Experts pour les questions se rapportant aux archives». La coopération intellectuelle, mars 1931, n³, p. 112-113.

26. - BONNET, Henri. L'œuvre de l'Institut International de Coopération Intellectuelle. Paris : Académie de droit international, 1938.

27. - «L'activité de l'Office International des Musées - Conclusions adoptées par la Conférence Internationale pour l'étude des méthodes Scientifiques Appliquées à l'Examen et à la Conservation des œuvres d'art, Rome, 13-17 octobre 1930 ». Mouseion, n¹3/14, 1931, p. 126-130.

28. - « Première Conférence Internationale pour la protection et la conservation des monuments d'art et d'histoire ». Bulletin de la Coopération Intellectuelle, n6, juin 1931, p. 290-291.

29. - « L'activité de l'OIM, septembre 1932-septembre 1933. Rapport à la CICI ». Mouseion, n²3/24, 1933, p. 247-248.

30. - « L'activité de l'OIM, septembre 1933-septembre 1934. Rapport à la CICI ». Mouseion, n²7/28, 1934, p. 289-295.

31. - «L'activité de l'Office International des Musées en 1931-1932». Mouseion, n²1/22, 1933, p. 274-276.

32. - FAYET-SCRIBE, Sylvie. Histoire de la documentation en France. Paris : CNRS-Éditions, 2000.

33. - BLANDIN, Patrick. De la protection de la nature au pilotage de la biodiversité. Versailles : Quæ, 2009.

34. - FOUNDOUKIDIS, Euripide. "L'œuvre internationale de Jules Destrée dans le domaine des arts ». Mouseion, n³3-34, 1936, p. 7-16.

35. - KOTT, Christina. Préserver l'art de l'ennemi ? Le patrimoine artistique en Belgique et en France occupées (1914-1918). Bruxelles : PIE Peter Lang, 2006.

36. - DESVALLÉES, Andrée. "Termes muséologiques de base ». Publics et Musées, n7, 1995, p. 142-143. Signalons qu'Euripide Foundoukidis n'a pas inventé la notion de "patrimoine commun de l'humanité » : on la trouve dès 1923 sous la plume du premier directeur de l'IICI, Julien Luchaire. LUCHAIRE, Julien. « Note sur le rôle du nouveau Comité national français de coopération intellectuelle ». Dans RENOLIET, Jean-Jacques. L'Unesco oubliée. La Société des Nations et la coopération intellectuelle (1919-1946). Paris : Publications de la Sorbonne, 1999, p. 40.

37. - « Le Congrès pour le Pacte Roerich ». Le Soir, Bruxelles, 16 septembre 1931.

38. - ARBELLOT, Simon. « Dans nos beaux châteaux ». Le Temps, 10 décembre 1933.

39. - DESTRÉE, Jules. « La guerre et les Monuments de l'Esprit ». Le Soir, Bruxelles, 31 mai 1931.

40. - Archives de l'OIM, OIM. VI. 26a. «La protection des monuments en temps de guerre». Octobre 1933.

41. - DUPIERREUX, Richard. « La Conférence du Pacte Roerich invite les puissances à adhérer à ce Pacte pour la protection des œuvres en temps de guerre». Comœdia, 29 septembre 1933. SCHKLAVER, Georges. "L'association pour le Pacte Roerich répond à l'office International des Musées ». Comodia, 25 décembre 1933.

42. - «L'Office International des Musées et la Protection des Monuments et Euvres d'Art en Espagne ». Mouseion, Supplément Mensuel, septembre-octobre 1936, p. 1-5. 
43. - VISSCHER, Charles de. «Rapport préliminaire présenté au Comité de Direction de l'office International des Musées, le 12 octobre $1936 »$. Mouseion, n³5/36, 1936, p. 177-184 et Vol 47/48, 1939, p. 167-175.

44. - SANCHEZ CANTON, Francisco Javier. « Les premières mesures de défense du Prado au cours de la guerre civile en Espagne ». Mouseion, n³9/40, 1937, p. 67-73.

45. - ESCHOLIER, Raymond. «La beauté de l'Espagne appartient au monde. Le monde doit la sauver ». Le Journal, 11 septembre 1936. « Un nouveau cri d'alarme ». Idem, 3 novembre 1936.

46. - ESCHOLIER, Raymond, HUISMAN, Georges, OJETTI, Ugo. Exposition de l'art italien de Cimabue à Tiepolo. Paris : Musée du Petit Palais, 1935.

47. - « Les artistes italiens au service de la propagande fasciste. Les dons d'œuvres italiennes aux musées français (1932-1936) - exposition La Box, ENSA Bourges, C. Fraixe, 21 janvier-22 février 2010 ». Le Journal des Arts, n³18, 5 février 2010.

48. - MICHAUD, Éric. Histoire de l'art. Une discipline à ses frontières. Paris : Hazan, 2005, p. 104-113.

49. - JULLIARD, Mayte Garcia. «Por amor al arte : motivo de todos y fatales consecuencias para algunos ». Dans CABAÑAS BRAVO, M., LOPEZ-YARTO, RINCON GARCIA, E., CONSEJO, W. Arte en Tiempos de Guerra. España : Superior de Investigaciones cientificas, 2009, p. 553-558.

50. - KENYON, Frederic. « La protection du patrimoine artistique en Espagne ». Mouseion, $n^{\circ} 37 / 38$, 1937, p. 183-192.

51. - « Au Louvre le Prado? Non! à l'Exposition ». L'CEuvre, 7 mai 1937. « Les chefs d'œuvre du Prado seront exposés au Louvre ». L'Ëuvre, 9 mai 1937. « Les chefs-d'œuvre du Prado, à Paris : les négociations ont abouti ». L'Euvre, 12 mai 1937.

52. - RENAU, José. «L'organisation de la Défense du patrimoine artistique et historique espagnol durant la guerre civile ». Mouseion, n³9/40, 1937, p. 65-66.

53. - « Les Études de l'Office International des Musées et la Protection des Monuments et CEuvres d'Art en temps de guerre ». Mouseion, Supplément Mensuel, avril 1938, p. 1-3.

54. - Archives de l'OIM, OIM. VI. 26.(3). « Note de service sur la question du sauvetage des œuvres d'art espagnoles », 24 janvier/2 février 1939.

55. - Archives de l'OIM, OIM. VI. 26.(3). « Conventions passées à Figueras », 3 février 1939.

56. - «Vigilance artistique ». Justice, 2 février 1939. "Les trésors artistiques espagnols sont arrivés au Perthus ». Ère Nouvelle, 4 février 1939. «Les Chefs-d'œuvre espagnols sont transportés en France ». L'CEuvre, 5 février 1939. "L'acheminement des trésors artistiques espagnols vers Genève ». Les Débats, 8 février 1939. "Les trésors de l'art espagnol sont à Genève ". Journal de Genève, 15 février 1939.

57. - Archives de l'OIM, OIM. VI. 26.(3). « Inventaire des Trésors d'art espagnols - Communiqué de Section de l'information de la SDN », 30 mars 1939.

58. - « L'exposition du Prado à Genève ». Mouseion, Supplément Mensuel, juin 1939, p. 7-8.

59. - JULLIARD, Mayte Garcia. « $1^{\mathrm{er}}$ juin-31 août 1939: L'été espagnol du musée d'art et d'histoire ». Hippocampe, ${ }^{\circ} 5$, juin 2010, p. 203-232.

60. - «Avant-Projet de Convention Internationale sur la Protection des Monuments et CEuvres d'Art au cours des Conflits Armés ». Mouseion, n47/48, 1939, p. 180-201.

61. - «La Protection des Monuments et CEuvres d’Art en Temps de Guerre ». Mouseion, n47/48, 1939.

62. - FOUNDOUKIDIS, Euripide. «Commentaire du Projet ». Mouseion, $n^{\circ} 47 / 48,1939$, p. 202-214.

63. - Argentine, Belgique, Bolivie, Brésil, Chine, Danemark, Estonie, Finlande, France, Haïti, Iran, Irlande, Lettonie, Lituanie, Luxembourg, Mexique, Monaco, Norvège, Panama, Paraguay, Pérou, Pologne, Roumanie, Suède, Uruguay, Yougoslavie.

64. - Union de l'Afrique du Sud, Australie, Canada, Chili, Égypte, Grèce, Guatemala, Irak et Siam.

65. - « Appel du Président Roosevelt adressé le $1^{\text {er }}$ septembre aux Gouvernements du RoyaumeUni, de la France, de l'Allemagne et de la Pologne - Réponses - Déclaration commune des 
Gouvernements de la France et du Royaume-Uni (3 septembre 1939) - Communiqué de l'Office International des Musées (4 septembre 1939)». Mouseion, Vol. 47/48, 1939, p. 222-228.

66. - « L'Office International des Musées ». Le journal des débats, 8 septembre 1939.

67. - « La mise en sûreté de nos Trésors d'Art ». Le Soir, Bruxelles, 30-VIII-1939. « La protection de nos richesses artistiques». Le Temps, Paris, 1-IX-1939. "Les œuvres d'art de nos musées sont mises en lieu sûr ». Le Progrès de Lyon, 1-IX-1939. « Comment sauver nos œuvres d'art ». Candide, 6-IX-1939. « Pour saluer nos Chefs d'œuvre ». L'indépendant Belge, 7-IX-1939. « La protection des œuvres d'art». L'indépendant Belge, 9-IX-1939. "L'évacuation du Louvre». L'Illustration, 16IX-1939. «Comment ont été mises à l'abri les collections des Musées Nationaux ». Le Temps, Paris, 20-IX-1939. "Nos Trésors d'art sont à l'abri - Une promenade aux musées royaux ». Le Soir, Bruxelles, 26-IX-1939.

68. - FOUNDOUKIDIS, Euripide. «Commentaire du Projet ». Mouseion, Vol 47/48, 1939, p. 203-214 ; Idem. : «La coopération intellectuelle dans le domaine des arts, de l'archéologie et de l'ethnologie au cours de l'année $1939 »$. Mouseion, n49/50, 1940, p. 215-230.

69. - Convention pour la protection des biens culturels en cas de conflit armé. La Haye, 14 mai 1954. FRANCIONI, Francesco. «Des biens culturels au patrimoine culturel : l'évolution dynamique d'un concept et de son extension ». L'action normative de l'UNESCO, Vol. I. UNESCO, Leiden : Martinus Nijhoff, 2007, p. 231-247.

70. - Les quatre membres fondateurs du Comité international du Bouclier bleu (CIBB) sont l'ICOM, l'ICOMOS, le CIA et l'IFLA : http://www.ancbs.org/cms.

\section{RÉSUMÉS}

Quelles leçons les acteurs de la conservation du patrimoine dans l'Entre-Deux-Guerres ont-ils tirées de la Grande Guerre? Cet article répond en rappelant comment ils héritèrent de la question de la protection du patrimoine dans les conflits armés, puis quels réseaux leur permirent de la poser et d'y répondre ; cette histoire constitue la préhistoire du Bouclier bleu. Il montre en particulier que c'est dans l'Entre-Deux-Guerres, sous l'égide de la Société des Nations, que les réseaux internationaux de conservation que nous connaissons aujourd'hui se sont constitués et que l'idée d'un patrimoine commun de l'humanité a pu émerger pour exorciser le souvenir de la Grande Guerre. Contre les conventionnalistes, qui pensaient qu'une loi suffirait à le protéger, les experts de l'office international des Musées rappelèrent qu'il fallait d'abord éduquer les peuples à son respect. Contre les nationalistes, qui voyaient dans l'héritage du passé l'expression du génie des peuples, ils expliquèrent que les grands chefs-d'œuvre de l'humanité sont d'abord les produits d'échanges culturels. À l'heure du repli communautaire et de l'ouverture des frontières, la double leçon qu'ils tirèrent de la Grande Guerre est toujours d'actualité.

What lessons did heritage curators of the inter-war period learn from the First World War? This article sets out to answer this question by looking at the existing state of the question of cultural property protection during armed conflict, and by looking too at the networks that offered reflection on this question. The story in fact constitutes the prehistory of the Blue shield. It shows how during the inter-war period, under the aegis of the Society of Nations, the international networks of heritage curatorship we know today came into being, and that the idea of a shared heritage for all of humanity emerged as a kind of antidote to the memory of the Great 
War. If the 'conventionalists' thought that a law should suffice, the experts at the International Office of Museums believed that first and foremost was the necessity to educate people to respect the heritage. Against nationalists who saw heritage in terms of the expression of a nation's genius, they explained that the great masterpieces of humanity were the result of cultural exchanges. Today, as frontiers are opened up but as communities are turning in on themselves, the double lessons learned from the First World War are still pertinent.

INDEX

Mots-clés : patrimoine, conservation, Office international des Musées, Comité international du Bouclier bleu

\section{AUTEUR}

\section{PIERRE LEVEAU}

Chercheur associé EA.4100 HiCSA/CRPBC (Paris 1 Panthéon-Sorbonne). Dr. philosophie (ED. 356, AMU) leveau.p@wanadoo.fr 